\title{
The influence of solvent representation on nuclear shielding calculations of protonation states of small biological molecules
}

\author{
Christina C. Roggatz ${ }^{1}$, Mark Lorch ${ }^{1}$ and David M. Benoit ${ }^{1,2 *}$ \\ ${ }^{1}$ Chemistry - School of Mathematics and Physical Sciences, University of Hull, Cottingham \\ Road, Hull, HU6 7RX, UK; \\ ${ }^{2}$ E.A. Milne Centre for Astrophysics \& G.W. Gray Centre for Advanced Materials, University of \\ Hull, Cottingham Road, Hull, HU6 7RX, UK \\ E-mail: roggatz@outlook.com; *D.Benoit@hull.ac.uk
}

\begin{abstract}
In this study, we assess the influence of solvation on the accuracy and reliability of isotropic nuclear magnetic shielding calculations for amino acids in comparison to experimental data. We focus particularly on the performance of solvation methods for different protonation states, as biological molecules occur almost exclusively in aqueous solution and are subject to protonation with $\mathrm{pH}$. We identify significant shortcomings of current implicit solvent models and present a hybrid solvation approach that improves agreement with experimental data by taking into account the presence of direct interactions between amino acid protonation state and water molecules.
\end{abstract}

\section{Introduction}

Amino acids are the central building blocks of life and involved in many biochemical processes. The nuclear magnetic resonance (NMR) characteristics of these small biomolecules have been found to be crucial for the assessment of ligand binding, in pharmacological studies, during the assignment of chemical shifts in complex systems and for conformational studies. ${ }^{1}$ The chemical shifts of amino acids and very small peptides can be used, for example, to serve as "random coil" baseline for protein structure investigations. ${ }^{2}$ Any systematic deviations from these "baseline shifts", which depend primarily on the identity of the amino acid side chain and conformation, can be used to identify secondary structures such as $\alpha$-helices or $\beta$-strands. ${ }^{2}$ Chemical shifts not only provide insight into the molecular conformation of the close surrounding of a nucleus but also indicate changes in their chemical environment. ${ }^{2,3}$ This makes shifts ideal parameters for the investigation of molecular processes. Changes in chemical shift can give information on neighbouring groups or amino acid sequences in a peptide or protein. ${ }^{4}$ Amino acid side chains are also key components during catalysis in enzymes or in binding pockets of receptors and changes in their chemical shifts can help to analyse reaction pathways and to indicate substrate binding. ${ }^{5}$ 
However, NMR spectroscopy has its limitations when it comes to investigations of the underlying mechanisms at the atomistic level. ${ }^{6}$ To counteract these limitations, studies often make use of computational simulations and modelling to gain more detailed insights (see for example Han et $a l .{ }^{7}$ or Roggatz et $a l .{ }^{8}$ ). Amino acids are therefore also increasingly studied with computational methods in attempts to optimise computer simulation programs and protocols.

In order to validate computational simulations, it is crucial to compare results obtained through modelling to the available experimental data. However, this requires a realistic calculation of chemical shift values. In practice, chemical shifts are measured as change of resonance frequency of a nucleus relative to a given standard, for example the same type of nucleus in tetramethylsilane (TMS). ${ }^{9}$ The resonance frequency of a nucleus, and therefore its chemical shift, directly depends on the magnetic field that the nucleus experiences. This differs for each nucleus due to shielding or deshielding effects caused by electrons surrounding the nucleus, which can be described by a nuclear shielding constant $\sigma_{n u c}$. This nuclear shielding constant can be calculated at quantum chemical level (see Mulder \& Filatov ${ }^{2}$ for an overview). The chemical shift of a given nucleus $(\delta)$ can then be derived from the calculated shielding constant $\sigma_{n u c}$ and the shielding constant of the same nucleus type in the standard reference compound $\left(\sigma_{r e f}\right)$ used in the NMR experiments (e.g. TMS): ${ }^{10}$

$$
\delta=\sigma_{\text {ref }}-\sigma_{n u c}
$$

The reference shielding value $\sigma_{\text {ref }}$ can be obtained by a separate calculation performed for the reference compound. As can be seen in Eqn. (1), the chemical shift of a nucleus $(\delta)$ is related to the negative of its nuclear shielding value $\left(\sigma_{n u c}\right)$ due to the historical custom of a reversed frequency scale. This means nuclei that are more shielded than the reference nucleus have lower chemical shifts and those less shielded have higher chemical shifts.

As biomolecules appear almost exclusively in aqueous solution, the contribution of solvation $\left(\sigma_{\text {solvent }}\right)$ is a major factor impacting on the value of the shielding of a nucleus $\left(\sigma_{n u c}\right)$. In addition, there are several interactions between the solvent and solute that play critical roles in stabilising conformations and mediating molecular processes. ${ }^{11}$

Buckingham et al. identified four different contributions to the shielding effect of solvent $\sigma_{\text {solvent }}:^{12}$

$$
\sigma_{\text {solvent }}=\sigma_{b}+\sigma_{a}+\sigma_{E}+\sigma_{w}
$$

which is composed of a long-range bulk diamagnetic susceptibility effect $\left(\sigma_{b}\right)$, the anisotropy in the molecular susceptibility of the solvent molecules close to the solute $\left(\sigma_{a}\right)$, a polar effect $\left(\sigma_{E}\right)$ and Van der Waals forces between solute and solvent molecules $\left(\sigma_{w}\right){ }^{12}$ While $\sigma_{a}$ is particularly important for solvents with large $\pi$ systems, the polar effect $\sigma_{E}$ and van der Waals forces $\sigma_{w}$ can be assumed to dominate in aqueous solutions ${ }^{13}$. The polar effect $\left(\sigma_{E}\right)$ is caused by the charge distribution in the solvent molecules leading to the formation of an electric field that perturbs the electronic structure of the solute. ${ }^{12}$ This clearly has a strong effect on the magnetic shielding at the nuclei. Interactions through hydrogen bonds can be seen as a special manifestation of the polar effect $\left(\sigma_{E}\right)$ and have been shown to significantly affect ${ }^{1} \mathrm{H}$ chemical shift values of those protons directly involved in the H-bond. ${ }^{14}$ Van der Waals forces between solute and solvent molecules $\left(\sigma_{w}\right)$ can also contribute significantly to the overall solvent shift.

In order to appropriately model solvent interactions, a number of different solvation methods has been developed ranging from periodic molecular dynamic simulations of the solvent to implicit, explicit or hybrid models in ab initio and DFT calculations. The implicit solvation model averages the effects of all solvent molecules around the solute ${ }^{15}$ and simplifies the interactions of the solute- 
solvent system by describing the solvent with a single dielectric constant. ${ }^{16}$ The solute is placed into a cavity of a continuous polarisable medium (= solvent) and the interaction of the solute with the surrounding field is calculated at the cavity boundaries ${ }^{15,16}$. Amongst the implicit solvent models are the well-known polarised continuum model $(\mathrm{PCM})^{15,17}$ and the conductor-like screening model (COSMO $)^{18}$. More refined models include the integral equation formalism $(\text { IEFPCM })^{19}$, the COSMO-RS (realistic solvents) ${ }^{20}$ and the reference interaction site model $(\text { RISM })^{21}$. These models are frequently used during NMR calculations. However, while implicit solvation remains a computationally inexpensive approach that captures the effect of bulk solvent $\left(\sigma_{b}\right)$ well and is particularly suitable for large solutes, it often neglects local effects within the first solvent shell such as strong hydrogen bonds. ${ }^{22}$ These local effects are better represented by explicit solvation, also referred to as microsolvation, ${ }^{22}$ where individual solvent molecules are placed around the solute ${ }^{23}$. The number of solvent molecules can be varied but is kept as low as possible, typically one to ten, ${ }^{23}$ to reduce computational costs. Solute molecules can also be specifically allocated to certain functional groups or atoms of the solute in larger systems. This leads to a more accurate representation of short-range interactions between solute and solvent, such as $\sigma_{E}$ and $\sigma_{w} \cdot{ }^{22}$ Yet this also poses the risk of forming additional interactions with the solute or have dangling $\mathrm{O}-$ $\mathrm{H}$ bonds and lone pairs that would not be present experimentally. ${ }^{22}$ Explicit solvation is more computationally costly than the use of implicit solvation models. A combination of both models creates a hybrid solvation model, where a cluster of the solute and a small number of explicit solvent molecules is placed into the implicit dielectric field. ${ }^{22}$ This model provides a promising cost-effective way of treating solvation in large solute-solvent systems with strong local effects. It has previously been called a combined discrete-continuum model, cluster-continuum model or implicit-explicit model. ${ }^{24}$ For small biomolecules in aqueous solution it can be assumed that both the bulk solvent as well as local effects play a significant role in shielding the nuclei. However, although numerous studies focus on solvent models in the context of NMR chemical shifts, those are mostly limited to organic molecules. A systematic investigation of the use of hybrid solvation models for amino acids is to our knowledge still lacking.

Apart from causing significant solvent-solute interactions, water can give rise to a third factor influencing NMR properties: protonation. This is an ubiquitous process in biology and the most common ionisation process in proteins. ${ }^{25}$ Ionisation and proton transfer play significant roles in electrostatic interactions, ligand recognition, protein folding, enzyme catalysis, membrane potentials and the energetics of cells. ${ }^{25}$ Depending on the $\mathrm{pH}$ of the aqueous solution different protonation states of the same amino acid can be present at the same time. Even within the human body, $\mathrm{pH}$ ranges from 1.5 in the stomach to 8 in the pancreas. ${ }^{26}$ It is therefore essential to investigate different protonation states of biological molecules. However, the focus of research to date has been on the protonation states present at physiological $\mathrm{pH}$, which refers to the well buffered blood $\mathrm{pH}$ of $7.4( \pm 0.05)^{26}$, and neglected other protonation states. Biomolecules and in particular the presence in different protonation states in solution have not been fully studied in the context of NMR chemical shifts apart from a single study on L-alanine ${ }^{27}$.

In this study, we investigate the effect of solvation on nuclear shielding calculations for small biomolecules. We focus on the protonation states of four different amino acids and the solvation of their ionisable groups by systematically investigating the number of water molecules per ionisable group within a hybrid solvation model required to match experimental data. In contrast to other studies, we investigate amino acids with different side chains and ionisable groups, include $\mathrm{H}_{\mathrm{N}}$ nuclei and compare our computational results directly with a consistent set of experimental data. We assess the influence of the implicit solvent method, the magnetic Hamiltonian and the hybrid solvation model performance on the accuracy and reliability of their computed isotropic 
nuclear magnetic shielding, hereafter also referred to as computed nuclear shielding. We use a computationally cheap, static DFT approach and focus on the interaction of the ionisable groups with water molecules at two different hybrid levels. This approach provides new systematic insights into the solvation of protonation states in direct comparison to our consistently obtained experimental NMR data. 


\section{Methods}

\subsection{Amino acid test set}

For this study, we use a test set of four amino acids: glycine, L-alanine, L-cysteine and L-serine. For each amino acid, we investigate all protonation states present in a $\mathrm{pH}$ range of $0-14$ (Fig. 1). For the calculations of the protonated states of glycine, L-serine and L-cysteine, the lowest energy conformation according to Balabin ${ }^{28}$ and Noguera et al. ${ }^{29}$ were rebuilt using AVOGADRO (Version 1.1.0) ${ }^{30}$ and optimised using the UFF force field ${ }^{31}$ and a steepest descent algorithm. The xyz coordinates of each preliminarily optimised molecule were then used as starting conformation for further geometry optimisation. The zwitterionic and deprotonated forms were based on the structure of the neutral form and hydrogen atoms were added or removed as necessary. For Lalanine, the optimised neutral conformation was taken from Godfrey et al. ${ }^{32}$ and de-/protonated forms were obtained by subtraction or addition of a proton to the neutral structure.<smiles>NCC(=O)[O-]</smiles>

Gly -<smiles>C[C@H](N)C(=O)[O-]</smiles>

Ala-<smiles>[O]CC(=O)[O-]</smiles>

Glyz<smiles>C[C@H](N)C(=O)O</smiles>

Alaz<smiles>O=C(O)C[18O]</smiles><smiles>C[C@H](N)C(=O)O</smiles>

$\mathrm{Ala}^{+}$<smiles>N[C@@H](CS)C(=O)[O-]</smiles><smiles>N[C@@H](CS)C(=O)[O-]</smiles>

Cys ${ }^{-}$

Cys

Cys $^{+}$<smiles>N[C@@H](CO)C(=O)[O-]</smiles>

Ser -<smiles>N[C@@H](CO)C(=O)[O-]</smiles>

$\operatorname{Ser}^{2}$<smiles>N[C@@H](CO)C(=O)O</smiles>

Ser $^{+}$

Fig. 1: Protonation states of the non-polar amino acids glycine and L-alanine and the polar amino acids L-cysteine and L-serine used in the test set. 


\subsection{Computations}

Geometry optimisations were performed using the PBE0 exchange correlation functional ${ }^{33}$ with a pc-2 basis set ${ }^{34-36}$. The RIJ-COSX approximation ${ }^{37}$ was used with a def2-TZVPP/J auxiliary basis $\operatorname{set}^{38}$ including D3 dispersion corrections following Grimme ${ }^{39,40}$. Isotropic nuclear magnetic shielding values of ${ }^{1} \mathrm{H}$ and ${ }^{13} \mathrm{C}$ nuclei were calculated at the PBE0/aug-pc-2 level of theory, using the RIJ-COSX approximation with a def2-TZVPP/J auxiliary basis set and the individual gauge for localised orbitals method (IGLO) ${ }^{41}$. All calculations were run in ORCA (Version 3.0.0) ${ }^{42}$ and the resulting nuclear shielding constants were expressed with a factor of $10^{-6}$ in comparison to experimentally determined chemical shifts (ppm).

The DFT functional PBE0 has been chosen as it is known to perform very well for NMR calculations, ${ }^{10,43}$ especially for nuclei with electrostatic interactions and in biological systems ${ }^{44}$. Single point energy calculations and geometry optimisations ${ }^{44-46}$ as well as nuclear shielding calculations performed with $\mathrm{PBE} 0^{44,46,47}$ were previously found to be in good agreement with experimental data. PBE0 is therefore amongst the most popular functionals used in NMR calculations. However, for the basis set we opted against the widely used valence triple- $\zeta$ Pople basis sets ${ }^{48,49}$ (see for example in Bachrach ${ }^{50}$ and Baggioli ${ }^{44}$ ). Instead we chose the pc- $n$ and augpc- $n$ basis sets developed specifically for DFT calculations by Jensen ${ }^{34}$ as they were reported to perform better than the Pople basis sets ${ }^{51}$. The aug-pc- $n$ set contains added diffuse functions, which substantially improve basis set convergence for molecular properties that depend on regions far from the nuclei, such as electric multipole moments and polarisabilities ${ }^{51}$ and was therefore used for the NMR nuclear shielding calculations. Basis sets designed specifically for the calculation of nuclear magnetic shielding constants, such as the aug-pcS-2 basis set ${ }^{51}$, could be expected to perform better than aug-pc-n basis sets due to the inclusion of an additional tight p-type function. This was shown to significantly reduce the basis set error in relation to the basis set limits. However, aug-pcS-n have been developed with a test set of small inorganic and organic molecules based on basis set limits ${ }^{51}$ and little benchmarking with respect to experimental data has been performed to date. In a comparative test, the aug-pc-2 basis set showed a higher accuracy and reliability (correlation of fit) at lower computational cost than the dedicated aug-pcS-2 basis set for our amino acid test set (implicit solvation for both nuclei based on the same starting geometries, see Supplementary Information Table T1). We therefore decided to use the aug-pc-2 basis set. The IGLO method was used as it was the only method available in the version of ORCA used in this study, however a comparison with a different magnetic Hamiltonian is also shown in section 3.2. The focus of this study is to investigate the interaction of the ionisable groups with water molecules at two different hybrid solvation levels using a computationally cheap, static DFT approach. Note that nuclear quantum effects and dynamics have been neglected but their potential influence is discussed in the discussion section.

\subsection{Experimental measurements of chemical shifts}

NMR samples were prepared for all four different amino acids from their aminoacetic acid forms (powder, $>99 \%$ purity level, 20mg, Sigma Ultra, Sigma-Aldrich, UK) in sodium phosphate buffer $\left(10 \mathrm{mM}\right.$, with $\left.10 \% \mathrm{D}_{2} \mathrm{O}\right)$ at a $100 \mathrm{mM}$ concentration and with TMS as internal standard. TMS was used to calibrate the experimental NMR spectra, as it is insensitive to the different $\mathrm{pH}$ conditions, and set at $0 \mathrm{ppm}$. For each amino acid samples of different $\mathrm{pH}$ were prepared in which the respective amino acid was mainly present in protonated, zwitterion, deprotonated or fully deprotonated form (Cys only) based on their literature $\mathrm{p} K_{a}$ values $^{52}$ (Gly: $\mathrm{pH} 1.12,6.51$ and 11.99; Ala: $\mathrm{pH}$ 0.95, 6.20 and 12.17; Ser: $\mathrm{pH} 0.87,5.35$ and 12.41; Cys: $\mathrm{pH} 1.07,4.89,9.19$ and 12.77). 
All zwitterion, deprotonated and fully deprotonated forms were present with a fraction of $>99.5 \%$ in the samples, except the deprotonated form of L-cysteine (86\%). The protonated forms were present at a fraction of $>95 \%$ (Gly, Ala, Ser) or $>87 \%$ (Cys). Averaging calculated shielding constants according to the fractions of the individual forms did not change results significantly due to the high proportions of the respective forms of interest.

NMR spectra were measured using a Bruker Avance II $500 \mathrm{MHz}$ spectrometer at 298K. Proton chemical shifts were determined using the WATERGATE method ${ }^{53}$ for water suppression and proton resonance was measured at $500 \mathrm{MHz}$. Proton chemical shifts were determined with a 1D sequence with water suppression using the 3-9-19 pulse sequence with gradients ${ }^{53,54}$ and 64 scans ${ }^{13} \mathrm{C}$ chemical shifts were measured with the $1 \mathrm{D}{ }^{13} \mathrm{C}$ sequence in decoupled mode. Spectra were processed using Topspin Version 2.0 (Bruker Instruments, Karlsruhe, Germany). All measured NMR shifts will be made available in an online public repository.

\subsection{Comparison of computed isotropic nuclear magnetic shielding and experimental chemical shift values}

Experimentally, only a single chemical shift could be obtained for protons of $\mathrm{CH}_{2}$, methyl and amino groups. This is due to the fact that the exchange rate (rotation) of the protons in these groups is fast compared to the small difference in nuclear frequency and leads to averaging over the system. ${ }^{55}$ To facilitate comparison to the measured data, the calculated nuclear shielding values of protons of these groups were therefore averaged.

Calculated shielding constants $\left(x\right.$-axis, $\left.10^{-6}\right)$ were plotted against experimental chemical shift values ( $y$-axis, ppm) and a least-square linear curve fit was performed with IGOR pro (Version 6.02, WaveMetrics, Inc. 1988-2007) using the linear function:

$$
\delta_{\text {exp }}=a+\left(b \cdot \sigma_{\text {calc }}\right)
$$

where $a$ is the intercept with the $y$-axis and $b$ the slope of the line. The quality of the least-squares fit was assessed using the Pearsons correlation coefficient, $\mathrm{R}^{2}$. This direct comparison using calculated nuclear shielding values helps to avoid offset errors ${ }^{1}$. It further allows a direct analysis of method accuracy without interference by error cancellation, which can be observed when chemical shift values are calculated as difference between a molecular and a standard nucleus (see Eqn. (3)). The slope of the correlation is expected to be close to -1 due to the inverse relationship between nuclear shielding and chemical shift values. The intercept with the $x$-axis should in theory represent the shielding value of the ${ }^{1} \mathrm{H}$ and ${ }^{13} \mathrm{C}$ nuclei for the reference substance TMS. Therefore, substituting the $x$ value of the linear curve fit function with the shielding value calculated for TMS should give 0 . Any deviation from 0 gives an indication of the error of the chosen calculation methods. In order to determine accuracy, the shielding constants of the ${ }^{1} \mathrm{H}$ and ${ }^{13} \mathrm{C}$ nuclei of TMS were calculated with the methods described above for the amino acid forms and with the respective solvation models. The calculated values for TMS were substituted into the linear curve fit function and the function was solved for $y$. The difference between the result of the linear function and the expected value of $0 \mathrm{ppm}$ was stated as accuracy. A linear correlation between computed and experimental data is performed to evaluate the results and our approach is very similar to that described by Nazarski et al. ${ }^{56}$. This approach can also provide linear scaling factors (see for example the CHEmical SHIft REpository ${ }^{57}$ ), which can be used in future studies to transform calculated nuclear magnetic shielding constants to better match measured data in order to validate structures or correctly assign chemical shifts. ${ }^{10,56}$ In our study, however, we used this 
linear correlation to compare the raw computed results to the experimental data directly and did not scale our results in any way.

\section{Results and Discussion}

\subsection{The importance of solvation: Gas phase vs. COSMO}

We first optimised the geometries of the test set both gas phase and in implicit solvent (water) using the $\mathrm{COSMO}^{20}$ approach implemented in ORCA. The nuclear shielding values of each geometry was then calculated as described in section 2.2. The results were plotted in comparison to experimental chemical shift values determined for the respective nuclei. Carbon nuclear shieldings $\left({ }^{13} \mathrm{C}\right.$ ) (Fig. 2A) were found to correlate better with experimental values by $0.05 \%$ when calculated with implicit COSMO solvation $\left(R^{2}=0.9990\right)$ compared to gas phase. The accuracy of the nuclear shielding calculation was improved significantly by COSMO solvation, enhancing it by $2.30 \mathrm{ppm}$ to an overall accuracy of $\pm 2.92 \mathrm{ppm}$.

For hydrogen nuclei ( ${ }^{1} \mathrm{H}$, protons) (Fig. $2 \mathrm{~B}$ ) calculated nuclear shielding values were found to correlate better with experimental values by $4.88 \%$ when calculated with implicit solvation (COSMO) compared to gas phase. The overall correlation, however, was very weak with $R^{2}=$ 0.1987 for COSMO solvation. The accuracy of the proton nuclear shielding calculation was improved significantly by implicit solvation with COSMO, enhancing it by $2.21 \mathrm{ppm}$ to an overall accuracy of $\pm 1.09 \mathrm{ppm}$.

These results clearly show that solvation matters and significantly improves nuclear shielding calculations to better match experimental values. This is consistent with recommendations given for NMR calculations in commonly used program suites like ORCA and Gaussian. ${ }^{42,58}$ For ${ }^{13} \mathrm{C}$ nuclei, the correlation between calculated and experimental values is very high and is only improved slightly by the implicit solvation, while the accuracy is improved by $56 \%$. The slope of the linear regression is close to $-1 \pm 0.05$, indicating a well-performing method. ${ }^{10}$ In contrast, the correlation for ${ }^{1} \mathrm{H}$ nuclei is very poor, even when using implicit solvation (COSMO). The accuracy of proton nuclear shielding calculations, however, was improved by $33 \%$ upon solvation. The slope of the linear regression differs significantly from the expected values of around -1 , indicating a high systematic error with the chosen method.

These findings contrast with the statements of Sousa et al., ${ }^{59}$ who concluded that conformation of amino acids optimised in the gas phase present a reasonable alternative to those optimised with implicit solvation. Our results indicate that this is not the case when the conformations are used for calculations of NMR parameters. During our optimisations in the gas phase, the amino acid conformers underwent proton relocation in zwitterionic protonation states (see Fig. S3 in Supplementary Information). The accurate representation of amino acids in their natural protonation states, however, is critical to gain biologically meaningful results that can be used in future simulations. The inclusion of solvation during the geometry optimisation process is therefore essential for realistic nuclear shielding calculations. 

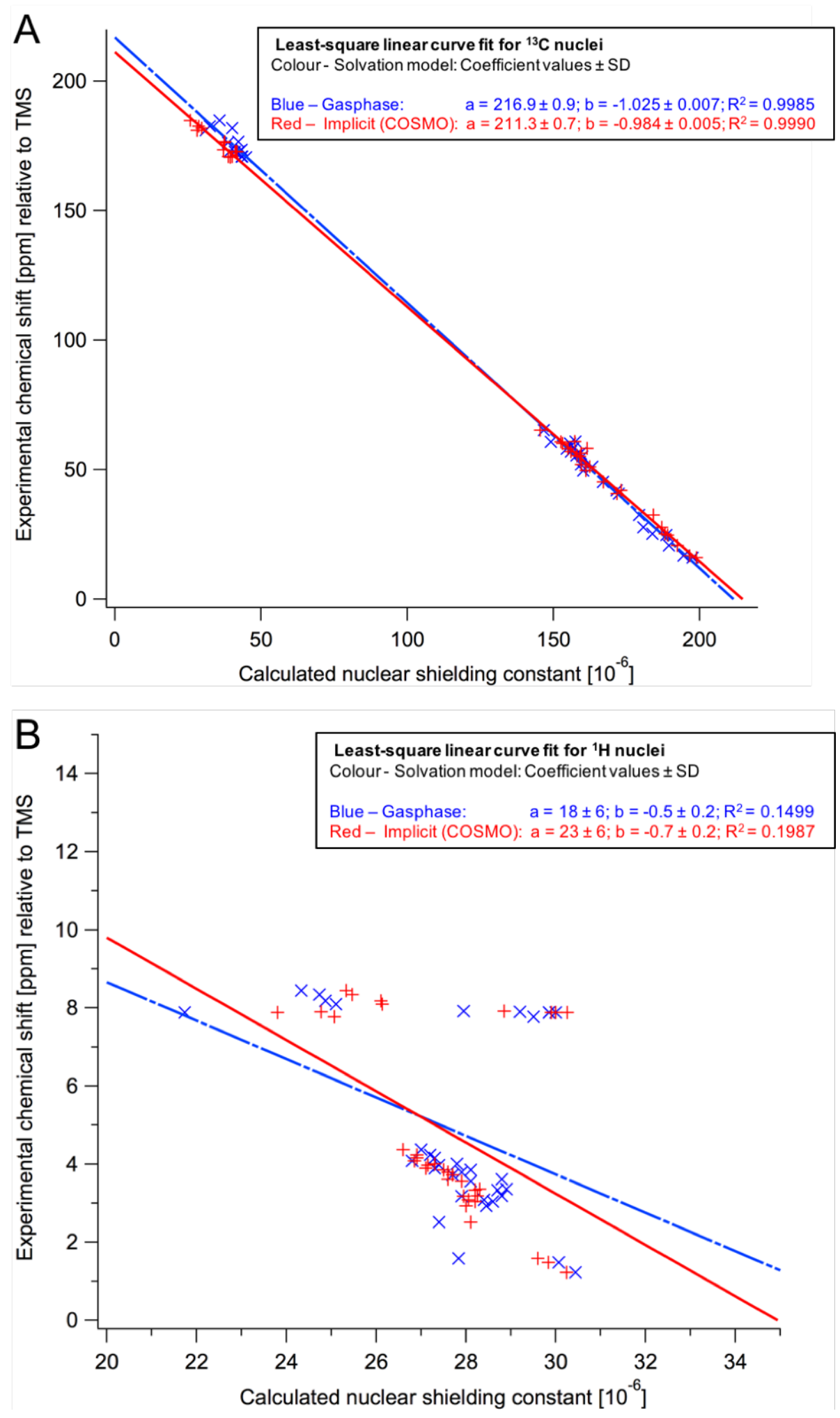

Fig. 2: Correlation between nuclear shielding values calculated in the gas phase or COSMO and experimental chemical shift values for ${ }^{13} \mathrm{C}(A)$ and ${ }^{1} \mathrm{H}(B)$ nuclei. For both nuclei types, calculations were performed in the gas phase (blue, intermitted line) and implicit solvation (COSMO) (red, continuous line). 


\subsection{Other implicit solvent models and the influence of the magnetic Hamiltonian}

After establishing the importance of solvation for one methodology, we wanted to ensure that our findings are also consistent across different implicit solvation models, such as the widely used IEFPCM ${ }^{19}$ implemented in Gaussian. We therefore calculated the nuclear shielding values of ${ }^{1} \mathrm{H}$ and ${ }^{13} \mathrm{C}$ with IEFPCM ${ }^{15,17}$ at the same PBE0/aug-pc-2 level of theory in Gaussian (Gaussian 09, Revision B.01 $)^{60}$ using the optimised geometries obtained from the ORCA calculations. While ORCA only implements the individual gauge for localised orbitals (IGLO) method ${ }^{41}$ for the magnetic Hamiltonian, the method to circumvent the so-called gauge problem implemented in Gaussian is the gauge-inducing atomic orbitals (GIAO) approach ${ }^{61,62}$. In order to obtain nuclear shielding constants independent from the magnetic field, both methods permit to mathematically cancel out the gauge origin by employing specific phase factors at atomic orbital (GIAO) or molecular orbital (IGLO) level. The effect of using GIAO instead of IGLO as magnetic Hamiltonian was evaluated by comparing the gas phase results obtained in this section and those of section 3.1 and is discussed below.

Carbon nuclear shieldings were found to correlate better with experimental values by $0.06 \%$ when calculated with IEFPCM solvation $\left(R^{2}=0.9988\right)$ compared to gas phase. The accuracy of the nuclear shielding calculation was improved significantly by IEFPCM solvation, enhancing it by $2.06 \mathrm{ppm}$ to an overall accuracy of $\pm 2.80 \mathrm{ppm}$. Proton shieldings were found to correlate better with experimental values by $2.59 \%$ when calculated with IEFPCM compared to gas phase. The overall correlation, however, was again very weak with $R^{2}=0.1886$ for IEFPCM solvation. The accuracy of the nuclear shielding calculation was improved by IEFPCM solvation, enhancing it by $0.16 \mathrm{ppm}$ to an overall accuracy of $\pm 3.06 \mathrm{ppm}$. The results for the two different implicit solvent methods (COSMO vs. IEFPCM) are very similar for ${ }^{13} \mathrm{C}$ nuclei when comparing the respective gas phase to implicit solvation results. For both solvation methods, slight changes to the correlation but clear improvements of the calculation accuracy are observed. For proton shieldings, both implicit solvent models show very poor correlation and when using IEFPCM solvation the accuracy is only improved slightly while in the COSMO calculation it is improved by a third.

The influence of the two different magnetic Hamiltonians, IGLO and GIAO, can be compared using the gas phase results. For both Hamiltonians the correlation between calculated and experimental data was very good for carbon nuclei and similarly poor for hydrogen nuclei. The accuracies for carbon differed by $0.3 \mathrm{ppm}(<6 \%)$ and for protons by less than $0.1 \mathrm{ppm}(<2.5 \%)$ between the two magnetic Hamiltonians. These differences are significantly smaller than the influence of implicit solvation. The choice of magnetic Hamiltonian is therefore of minor importance, which matches results of Facelli who reported calculations with sufficiently large basis sets to converge to the same nuclear shielding values with both methods ${ }^{9}$.

In comparison to previously reported accuracies, which typically range between 1.5 and 8 ppm for ${ }^{13} \mathrm{C}^{10,63,64}$, the accuracy of our approach with implicit solvation is relatively high with $\pm 2.92 \mathrm{ppm}$ $(\mathrm{COSMO})$ and $\pm 2.80 \mathrm{ppm}$ (IEFPCM). Common accuracies for proton shielding calculations are in the region of 0.1 to $0.4 \mathrm{ppm}^{10}$. This is significantly better than $\pm 1.09 \mathrm{ppm}$ (COSMO) and $\pm 3.06 \mathrm{ppm}$ (IEFPCM) obtained in our calculations. Frank et al. stated that the main reason for errors in proton chemical shifts is the neglect of explicit solvent molecules. ${ }^{1}$ Proton chemical shifts are known to be highly influenced by their environment and H-bonds with solvent molecules can significantly alter their shielding by giving rise to the polar shielding effect $\left(\sigma_{E}\right)$ (see Eqn. (2)). We therefore went on to test the effect of additional explicit solvent molecules on the nuclear shielding calculation. 


\subsection{Hybrid models}

In order to improve the performance of our model for protons, we investigated how adding explicit water molecules affects the correlation and agreement of nuclear shielding constant calculations with experimental data. For this purpose, we created two types of hybrid solvation models by adding either one water molecule per ionisable functional group of the solute (low level hybrid, LH) or one water molecule per ionisable proton of the solute (high level hybrid, HH) (see Fig. 3). For the low hybrid model, water molecules were added directly next to the functional groups of the solute using AVOGADRO and the geometry optimisation and nuclear shielding constant calculations were performed as described in section 2.2 with COSMO as implicit solvation. For the high hybrid model, a two-step process was applied to ensure the saturation of all protons with a respective water molecule. First, water molecules were added directly next to each ionisable proton of the solute using AVOGADRO, followed by a geometry optimisation at HF-3C level of theory ${ }^{65}$. HF-3C is a corrected small basis set Hartree-Fock method and is specifically developed for the fast computation of structures and non-covalent interactions in large molecular systems ${ }^{65}$. Optimised geometries were checked for saturation and, if required, more water molecules were added followed by another geometry optimisation at HF-3C level of theory until all ionisable protons were saturated with an $\mathrm{H}$-bond from a water molecule. Then a full geometry optimisation and nuclear shielding constant calculation were performed as described in section 2.2 with COSMO as implicit solvation. For the calculation of TMS with the high hybrid solvation model, a symmetry approach was used and the nuclear shieldings for only one ${ }^{13} \mathrm{C}$ and three ${ }^{1} \mathrm{H}$ nuclei were calculated upon saturation with hydrogen bonds to explicit water molecules.

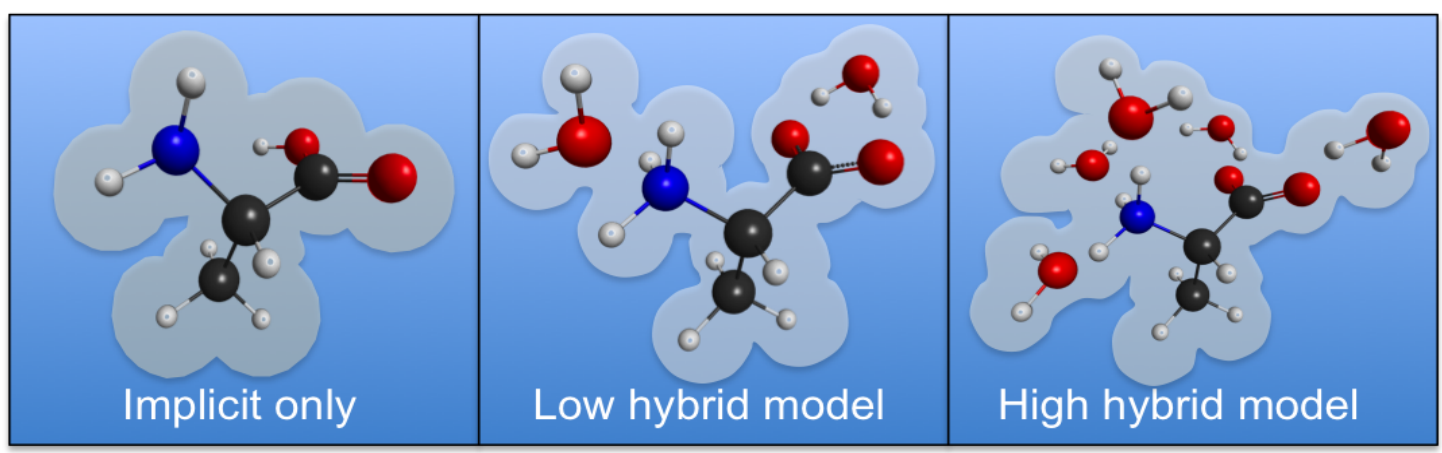

Fig. 3: Schematic presentation of the different solvation models for L-alanine: implicit (left), low hybrid (LH, center) with one water molecule per ionisable group and high hybrid (HH, right) with one water molecule per ionisable proton.

For carbon nuclei (Fig. 4A) the correlation with experimental values was only very marginally affected when calculated with low hybrid solvation $\left(R^{2}=0.9994\right)$ or high hybrid solvation $\left(R^{2}=\right.$ $0.9989)$ compared to implicit solvation only $\left(R^{2}=0.9990\right)$. The accuracy of the nuclear shielding calculation was clearly higher $( \pm 2.48 \mathrm{ppm})$ for the low hybrid solvation than for calculations with implicit solvation only. For high hybrid solvation model the accuracy however was much lower ( $\pm 5.5 \mathrm{ppm})$.

For protons (Fig. 4B), the correlation to experimental data could be significantly improved by $13.03 \%$ for low hybrid and by $25.84 \%$ for high hybrid solvation compared to implicit solvation only. In contrast, the accuracy of the nuclear shielding calculations with hybrid models was lower than with the implicit model. In all three solvation models a group of clear outliers from the expected linear correlation could be observed. 

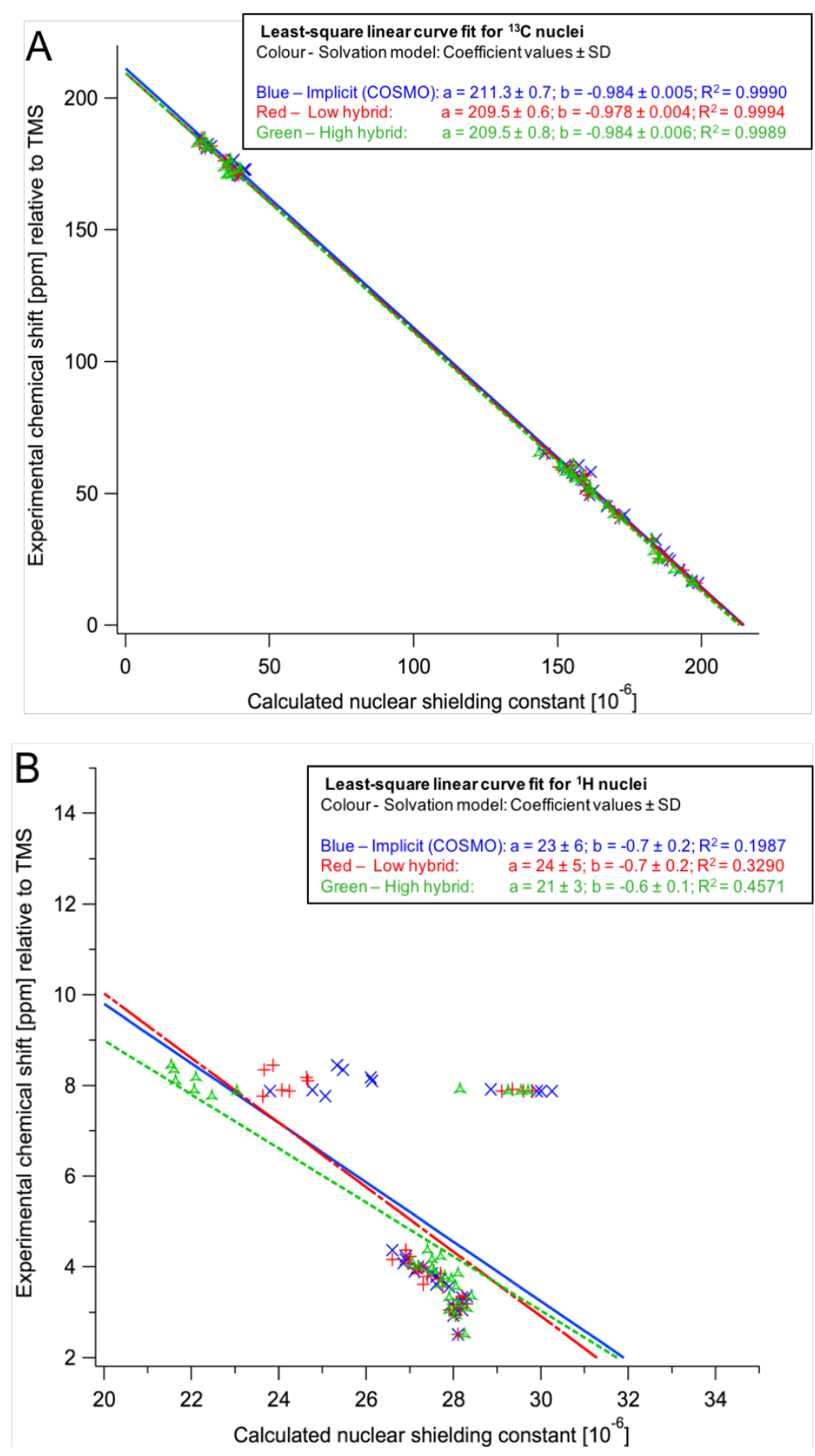

Fig.4: Correlation between nuclear shielding values calculated in implicit, low or high hybrid solvation and experimental chemical shift values for ${ }^{13} \mathrm{C}(A)$ and ${ }^{1} \mathrm{H}(B)$ nuclei. For both nuclei types calculations were performed with implicit solvation (COSMO) (blue, continuous line), low hybrid solvation (red, intermitted line) and high hybrid solvation (green, dotted line). 
Protons bound to carbon atoms $\left(\mathrm{H}_{\mathrm{C}}\right)$ were found to show the expected linear correlation. In contrast, protons bound to nitrogen $\left(\mathrm{H}_{\mathrm{N}}\right)$ did not show the expected correlation due to the outliers with shielding values of around $30 \times 10^{-6}$ (Fig. 5). These outliers were identified to originate from calculated shielding constants of protons in amino groups of the deprotonated amino acid states. A closer look at the optimised geometries revealed that in all (fully) deprotonated states the nitrogen of the amine group formed a hydrogen bond with a water molecule, acting as a hydrogen bond acceptor. In all zwitterionic and protonated forms, however, the hydrogen atoms of this amino group formed hydrogen bonds with the surrounding water, acting as H-bond donors. These hydrogen bonds between amino group $\mathrm{H}_{\mathrm{N}}$ and oxygen of water molecules clearly improved the correlation with experimental data by "shifting" the calculated values to the left compared to implicit solvation with no explicit water molecules (= no H-bonds). Indeed, the successive shift from $\mathrm{H}_{\mathrm{N}}$ values in blue, red and green to the left can be seen in Fig. 4B.

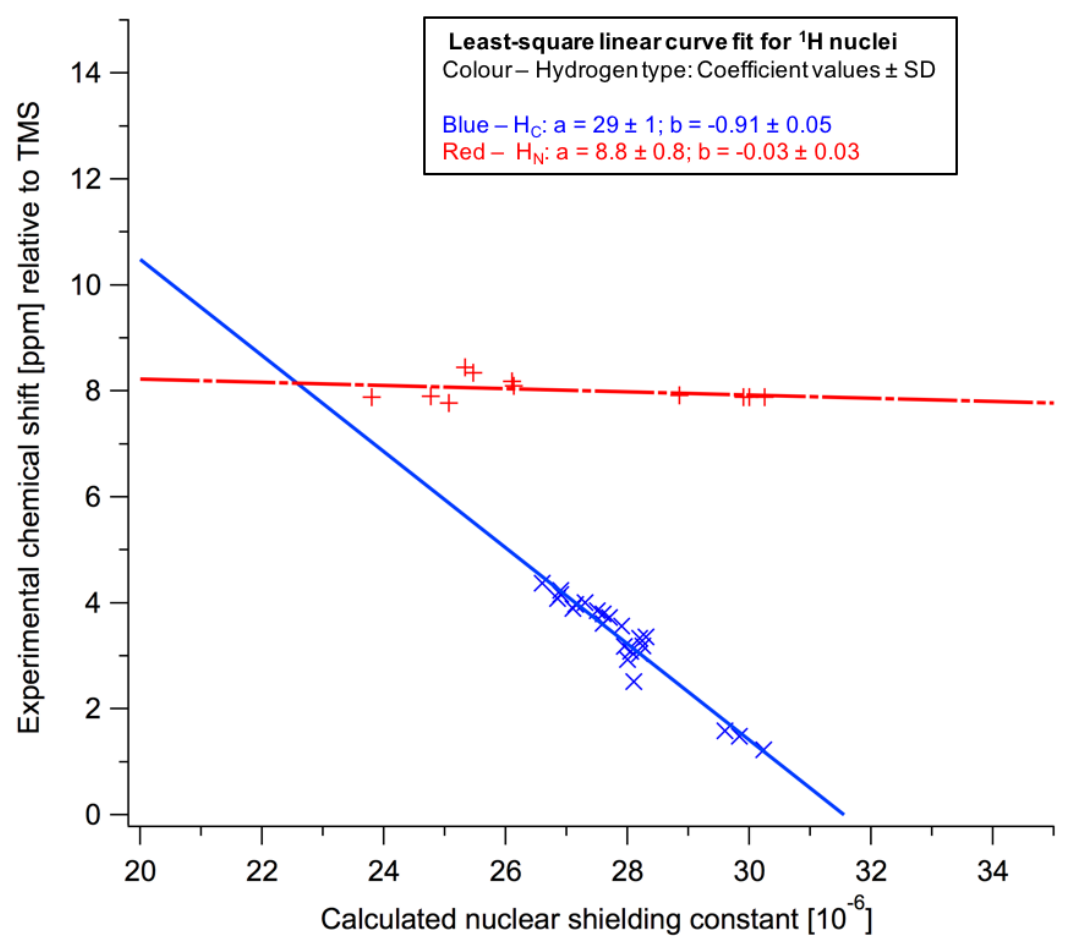

Fig. 5: Comparison between correlation of ${ }^{1} \mathrm{H}$ nuclei attached to carbon (blue, continuous line) or nitrogen (red, intermitted line) calculated with COSMO solvent.

In order to investigate why deprotonated amino acid states wouldn't form the same hydrogen bonds in more detail, we chose the glycine anion as an example. We found that the partial charge at the amino group $\mathrm{H}_{\mathrm{N}}$ in the anion was significantly lower than in the zwitterionic and protonated state (see Supplementary Information, Fig. S4). This could be caused by the overall negative charge of the molecule due to the deprotonated carboxyl group. This seems to cause a Coloumb repulsion between the amino $\mathrm{H}_{\mathrm{N}}$ and the oxygen of water and so make the interaction of the $\mathrm{H}_{\mathrm{N}}$ with water less favourable. We were able to optimise the system at a $\mathrm{H}_{\mathrm{N}}$-water distance of $2.4 \AA$ (see Supplementary Information, Fig. S5), which corresponds to a weak hydrogen bond ${ }^{66}$ and yields a nuclear shielding constant of $29 \times 10^{-6}$. We also observed that when the $\mathrm{H}_{\mathrm{N}}$ of anionic glycine were constrained at a given distance to a hydrogen-bond acceptor, such as the oxygen atom of a 
water molecule, their nuclear shielding values are affected proportionally to the distance (Supplementary Information, Fig. S6). This has been previously also observed by Moon \& Case ${ }^{67}$. Based on the fitted correlation curve in Fig. 5, we would expect $\mathrm{H}_{\mathrm{N}}$ shielding constants of around $25 \times 10^{-6}$, which would correspond to a distance of $1.7-1.8 \AA$. The DFT methodology chosen in this study was found to be not suitable to correctly calculate this hydrogen bond interaction in deprotonated protonation states because electrostatic forces seem to overpower the forces of the hydrogen bonds. D3 is known to have issues with charged systems and using the recently developed D4 dispersion interactions ${ }^{68}$ in future studies will potentially address this. Here, we therefore excluded the values of amino $\mathrm{H}_{\mathrm{N}}$ of deprotonated states in the following.

Analysing the results for proton nuclear shielding constants after excluding the outliers revealed a much clearer picture as shown in Fig. 6. The addition of one water molecule per ionisable functional group in the low hybrid model improved the correlation to experimental data by $11.8 \%$ and the accuracy by $\pm 1.00 \mathrm{ppm}$ to $\pm 0.81 \mathrm{ppm}$ compared to implicit solvation. With the high hybrid model, correlation was improved by $13.0 \%$ and accuracy by $\pm 0.88 \mathrm{ppm}$ compared to implicit solvation. However, the low hybrid solvation model performed best with regards to accuracy. The use of hybrid solvation shows a clear improvement to the nuclear shielding calculations.

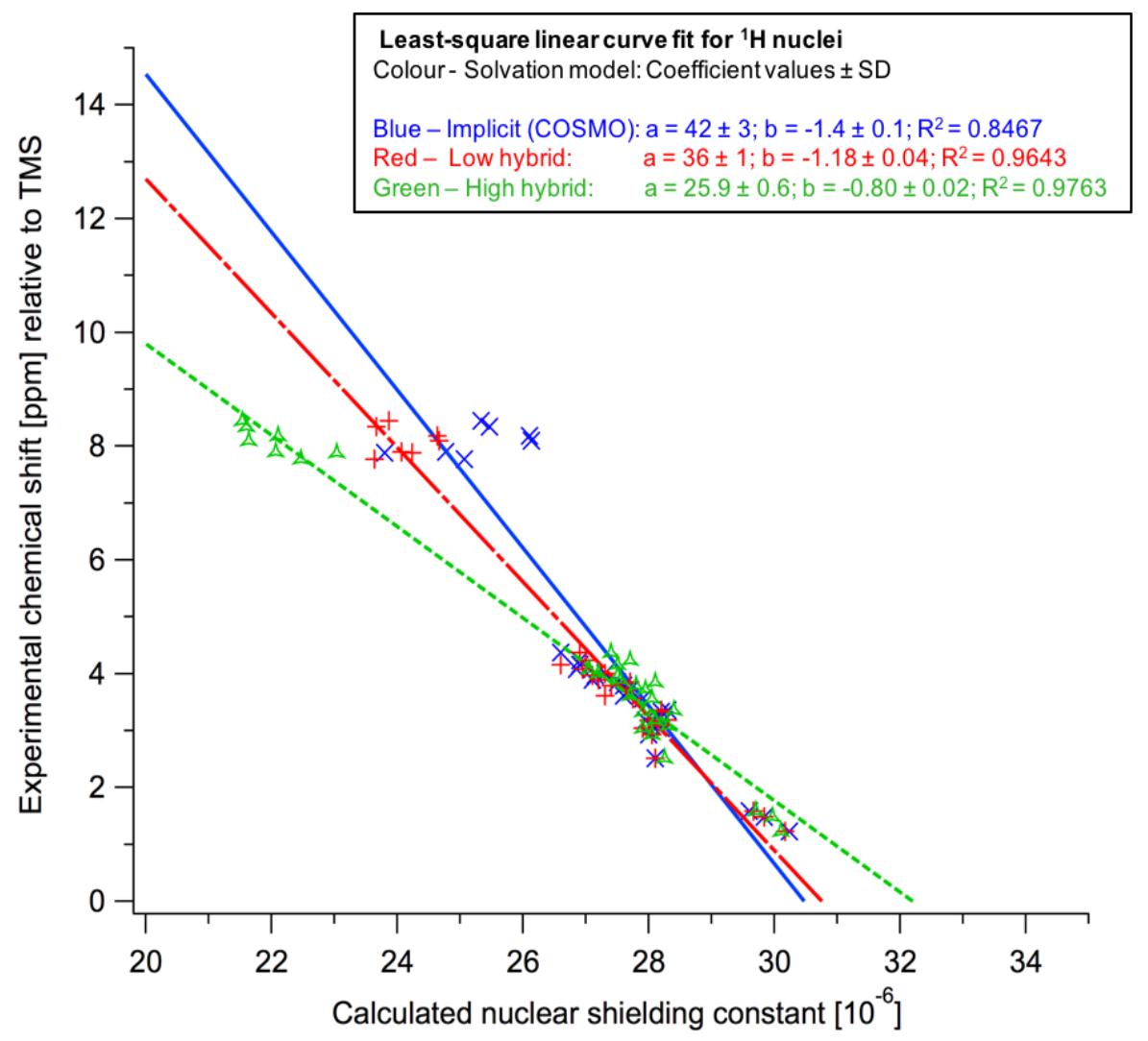

Fig. 6: Correlation between calculated nuclear shielding values and experimental chemical shift values for ${ }^{1} H$ nuclei excluding protons of the amino groups in anions. Calculations were performed with implicit solvation (COSMO) (blue, continuous line), low hybrid solvation (red, intermitted line) and high hybrid solvation (green, dotted line). 


\subsection{Discussion}

In this paper we illustrate the importance of solvation and the impact of different solvation models in the context of nuclear shielding calculations. It was established that the inclusion of solvent is crucial to obtain more accurate nuclear shielding predictions, irrespective of the implicit solvation model or program used. The addition of explicit solvent molecules to achieve a more accurate solvent representation is essential to represent the direct solute-solvent interactions like hydrogen bonds. It significantly improves calculated nuclear shielding values, especially those of protons. Using on average one water molecule per ionisable group in a hybrid approach was found to be sufficient.

\section{Performance of the hybrid models}

The low hybrid solvation model was found to perform best. It showed higher accuracies than the high hybrid solvation model in all cases and only minor differences in correlation. This contradicted our expectations because experimental and theoretical investigations on gas phase hydration show that for stabilisation of the zwitterionic form of non-polar amino acids, for example, 5 or more water molecules are required. ${ }^{69-73}$ Dračínský and Bouř reported for the zwitterionic form of L-alanine that a cluster of the solute and the first hydration shell in combination with PCM implicit solvation yields the closest results in comparison to experimentally observed differences between vacuum and solution ${ }^{27}$. The worse performance of the high hybrid model in our study seems to be caused by an overrepresentation of the solute-solvent interaction at the amino terminus. This significantly influences the linear fit and accuracy by affecting the slope as the amino $\mathrm{H}_{\mathrm{N}}$ nuclei are located at the top end of the graph. A similar observation of overestimation has also been reported by Cossi \& Crescenzi for ${ }^{17} \mathrm{O}$ shieldings of small organic molecules when optimised in water clusters. ${ }^{13}$ The averaged shielding values of the hydrogen atoms of the amino group significantly determine the slope of the regression and therefore the accuracy. Potentially the addition of three water molecules at the amino terminus in combination with the implicit solvation does not represent a realistic situation. Results of Panuszko et al. suggest an average number of 2.6 -2.7 water molecules per amino group to form direct interactions in hydration. ${ }^{70}$ This means that not all hydrogen atoms in the amino group are saturated by water molecules at all times. The lower number of water molecules used in the low hybrid model might therefore explain why it is performing better. For polar amino acids $1-4$ water molecules were found to be sufficient to stabilise the zwitterionic form in gas phase hydration. ${ }^{69}$ In a wide range of protein crystal structures the amino acid:water ratio was determined as 0.4 to 2.7 depending on the polarity and solvent accessibility of the amino acid in a given protein conformation. ${ }^{74}$ Therefore one water molecule per titratable group would present a suitable, more realistic and computationally less expensive approach for peptides, too.

\section{Method performance differs with nucleus type}

The performance of computational methods in comparison to the experimental data can be evaluated based on the slope of the linear regression between experimental and calculated data. This slope should be within a range of $-1.00 \pm 0.05$ in order to indicate only minimal systematic error. ${ }^{10}$ The set of computational methods chosen here were found to perform very well for carbon nuclei as the regression slopes of all solvation models were $-1.00 \pm 0.02$. Systematic errors causing a change in the slope and therefore requiring significant scaling were not apparent. In contrast, for proton shieldings the slope values obtained for this test set deviated by up to $20 \%$ from the expected -1.00 when hybrid solvation models are used. This indicates the presence of a systematic error, even when the identified outliers are excluded. However, it has to be stressed that, in contrast to most other published studies, here we also included the nuclear shielding values of 
protons in amine groups $\left(\mathrm{H}_{\mathrm{N}}\right)$ and do not apply scaling factors. These protons are known to be very sensitive to molecular geometry and the formation of hydrogen bonds. ${ }^{75-77}$ Therefore they provide lots of information and play a significant role in determining the regression parameters, as already discussed above. However, they have often been excluded in studies, ${ }^{1}$ partly also due to experimental difficulties in their determination and their particular dependence on the solvent used. ${ }^{78}$ In our study, the $\mathrm{H}_{\mathrm{N}}$ shieldings of protonated and zwitterionic amino acid forms were found to correlate reasonably well with the experimental data that could be obtained for 29 of 35 protonation states. Including the direct solute-solvent hydrogen-bond in the low hybrid model significantly improved the accuracy by decreasing the average shielding of the amino group protons. The inclusion of explicit water molecules was also reported to improve the agreement between calculated and experimental solution-phase NMR data of the amino ${ }^{1} \mathrm{H}$ chemical shift in guanine. ${ }^{76}$ Exner et al. also documented that the inclusion of explicit solvent can significantly increase the chemical shift of $\mathrm{H}_{\mathrm{N}}$ by $2.34( \pm 1.2) \mathrm{ppm},{ }^{79}$ which is comparable to the changes observed in our study with $1.5( \pm 1.2)$ ppm.

\section{Reliability and accuracy in comparison to other approaches}

A chosen computational method always aims to strike a suitable balance between reliability and validity with the lowest computational costs possible. Our chosen method was found to provide reliable results for carbon and hydrogen nuclei including $\mathrm{H}_{\mathrm{N}}$, when the low hybrid approach is used, with overall very high correlation coefficients $\left(\mathrm{R}^{2}\right)$ of $\geq 95 \%$. Besides its reliability for amino acids, it was further found to achieve good accuracies of carbon and proton nuclear shielding calculations in comparison to literature values in general. For carbon nuclei the accuracies reported in the literature range from $\pm 5 \mathrm{ppm}$ to $\pm 1.53 \mathrm{ppm}^{1,63,64,80,81}$ depending on the level of theory and the nature of the dataset they are compared to. Best accuracies were reported for an approach using $\operatorname{CCSD}(\mathrm{T})$ with a large basis set and accounting for vibrational effects for a set of very small molecules in the gas phase. ${ }^{81}$ With DFT functionals the best accuracies for carbon nuclear shielding compared to experiment were reported by Frank et al. for the 32 amino acid long HA2 domain of the influenza virus glycoprotein hemagglutinin with $\pm 1.53 \mathrm{ppm}(\mathrm{mPW} 1 \mathrm{PW} 91 / 6-311 \mathrm{G}(\mathrm{d})$ with PCM and point charges in environment). ${ }^{1}$ The same method yielded accuracies of \pm 3.44 and $\pm 2.51 \mathrm{ppm}$ for other parts of the same molecule. ${ }^{1}$ These accuracies are similar to the values reported recently by Benassi, who tested a number of DFT functionals and noted WP04/DGTZVP amongst the best performing DFT functionals for a test set of organic molecules with an accuracy of $\pm 3.68 \mathrm{ppm} .{ }^{43}$ The accuracy of \pm 2.5 obtained for carbon nuclear shielding in our study (PBE0/aug-pc2) is therefore very good, albeit for a small data set of four amino acids. Hydrogen nuclear shieldings are reported with accuracies of between $\pm 0.86 \mathrm{ppm}$ and $\pm 0.2 \mathrm{ppm}$ in the literature. ${ }^{1,80,82}$ The best accuracy obtained for protons most recently reported was $\pm 0.11 \mathrm{ppm}$, calculated with the WP04 functional (specific for chloroform solvation) and a $6-311+\mathrm{G}(2 \mathrm{~d}, \mathrm{p})$ basis set by Benassi for a test set of organic molecules with solvation in chloroform. ${ }^{43}$ The PBE0 functional with the same basis set was found to perform almost equally well with an accuracy of $\pm 0.12 \mathrm{ppm}$ in the same study. ${ }^{43}$ This is a higher accuracy than we obtained in this study, but using our low hybrid solvation model we achieved an accuracy in the range reported by literature. This is very good considering the fact that, in contrast to the cited studies, our test set contains different protonation states and therefore differently charged molecules. It further includes not only the shieldings of protons attached to carbon but also to nitrogen atoms, which are usually excluded. 


\section{The importance of test set nature and a consistent experimental data set}

The quality of the experimental data used for comparison is besides the computational methods the most crucial factor to obtain a realistic estimation of accuracy. It can profoundly affect the determined accuracy in two ways. Firstly, the type and diversity of molecules and molecular protonation states included in the test set determines how representative the obtained accuracy is for any other given molecule. The accuracy obtained for a test set with small organic, mostly rigid molecules in the gas phase is unlikely comparable to the accuracy obtainable for nuclear shieldings of a test set with more complex, flexible biomolecules in aqueous solution. This is illustrated by the range of accuracies obtained with similar computational methods for different test sets (e.g. comparing the benchmarking of Flaig et al. ${ }^{80}$ and Benassi ${ }^{43}$ ). Secondly, the experimental data itself can contain variabilities in the chemical shift values due to differences in NMR equipment, temperature, solvent, concentration or reference compound and referencing methods. ${ }^{78,83,84}$ However, there are very few consistent experimental data sets with the same or at least comparable physicochemical factors, such as temperature and concentration.

The accuracy values reported in the literature are always only representative of their particular test set, making a direct comparison difficult. In many cases the test sets are dominated by small organic molecules, for example in the studies of Rablen ${ }^{82}$ or Auer et al. ${ }^{81}$. Furthermore, most computational results are compared to experimental data obtained by different working groups at different conditions. This is inevitably the case for large test sets containing a large number of compounds (e.g. in Benassi's study ${ }^{43}$ ). Our consistent, high quality experimental data set with an accuracy of $\pm 0.001 \mathrm{ppm}$ for proton and $\pm 0.002 \mathrm{ppm}$ for carbon shifts obtained with constant temperatures and consistent concentrations across samples in the same solvent with the same reference compound minimises the influence of variations in the experimental data on the analysis of shielding accuracies. It allows us to obtain a very good picture of the accuracy of the calculated shielding values alone. The results for this test set can only be compared indirectly to other approaches as biomolecules have only recently been considered in NMR test sets and amino acids are often not included. Our methodological approach and the obtained accuracies for a test set focussing on amino acid protonation states therefore provides a meaningful benchmark of particular importance for studies on amino acids, peptides and proteins.

\section{The indirect solvent effect, conformational sampling and nuclear quantum effects}

Apart from the direct and obvious effects of hydrogen bond formation and changes to the electrostatic environment, explicit solvent can also have an indirect effect that can influence the calculated shieldings: conformational change. As Cossi \& Crescenzi showed very clearly in their study of small organic molecules in aqueous solution, the direct solvent effect can be well separated from an indirect effect caused by the water molecules altering the molecular conformation of the solute with approximately $10 \%$ the size of the direct solvent effect. ${ }^{13}$ However, Monajjemi et $a l$. reported in their study that the solvent-induced shielding variation is more likely influenced by the intensity of the solvent reaction field than the molecular geometry induced by the solvent. ${ }^{85}$ Degtyarenko et al. concluded from a molecular dynamics (MD) study of L-alanine in aqueous solution that the first hydration shell of amino acids is localised around the carboxylate and ammonium functional groups. ${ }^{86}$ This shell is highly ordered and quite rigid but the participating water molecules were found to constantly exchange. ${ }^{86}$ This would agree with our findings for the hybrid models. In order to represent the varying solute-water interactions better, conformational averaging would be a good potential technique. Its importance has been increasingly highlighted over the past years. ${ }^{87-89}$ Results of Kwan et al. and Exner et al. suggest that averaging over MD snapshots for which the chemical shift values are calculated individually yields a significant improvement of accuracy. ${ }^{79,87}$ The use of only one optimised lowest energy conformer for each 
protonation state in our study will therefore have to be compared to results obtained with methods using MD and conformational averaging (for example as described by Dračínský et al. ${ }^{89}$ ) in the future. This could also help to resolve the problems observed for $\mathrm{H}_{\mathrm{N}}$ nuclei in deprotonated amino acid states. An additional factor to be considered in this context is the influence of nuclear quantum effects (NQEs), such as zero-point vibrations and tunnelling, ${ }^{90,91}$ which have been neglected in our study in order to reduce computational cost. NQEs can play a significant role, particularly in hydrogen-bonded systems, due to the low mass and thus high delocalisation of protons. ${ }^{90}$ A proton can be slightly shared or fully delocalized between donor and acceptor depending on the strength of the hydrogen bond. ${ }^{90}$ These delocalisation effects vary with temperature but also affect nuclear shielding. ${ }^{92}$ Path integral molecular dynamics (PIMD) simulations have shown that including NQEs result in a better correlation between calculated and experimental chemical shifts. ${ }^{91}$ Indeed, Dračínský and Hodgkinson showed that the ${ }^{13} \mathrm{C}$ shieldings decrease by more than $5 \mathrm{ppm}$ in glycine crystals and by more than 9 ppm in L-alanine crystals upon inclusion of NQEs - depending on the position and number of hydrogen atoms attached to the nucleus. ${ }^{91}$ For ${ }^{1} \mathrm{H}$ shieldings, they observed a decrease between 0.4 and 1.2 ppm for crystals of glycine and L-alanine, respectively. ${ }^{91}$ Considering the importance of hydrogen-bonds for accurate and reliable calculations of nuclear shielding in solution, as illustrated in our study and our observations regarding the anionic $\mathrm{H}_{\mathrm{N}}$, the inclusion of NQEs in future studies could be an important addition to our approach.

\section{Conclusion}

This study highlights that the inclusion of solvent is crucial to obtain more accurate nuclear shielding predictions, irrespective of the implicit solvation model or program used. The addition of explicit solvent molecules to achieve a more realistic representation is essential to account for the direct solute-solvent interactions such as hydrogen bonds. It significantly improves calculated nuclear shielding values, especially those of protons. Using on average one water molecule per ionisable group in a hybrid approach was found to be sufficient to achieve a good accuracy.

Nevertheless, the use of a single optimised lowest energy conformer for each protonation state in our study does not allow any conformational averaging. Future work should compare this approach to results obtained with methods using MD and conformational averaging as this could also help resolve the issues observed for $\mathrm{H}_{\mathrm{N}}$ nuclei in deprotonated amino acid states.

In contrast to other studies, our test set contains different protonation states and therefore differently charged molecules. It further includes not only the shieldings of protons attached to carbon but also to nitrogen atoms, which are usually excluded. Moreover, it provides an exceptionally consistent experimental data set for comparison (to be released later). Our study suggests that using one explicit water molecule per titratable group would present a suitable, realistic and computationally inexpensive approach to determine NMR shielding for peptides.

\section{Acknowledgments}

We acknowledge the Viper High Performance Computing facility of the University of Hull and its support team as well as funding for CCR's PhD scholarship by the University of Hull. 


\section{Supplementary Information}

An additional table comparing aug-pc-2 and aug-pcS-2 basis set performance (T1), a figure showing the results for calculations in Gaussian (S2) and figures illustrating the characteristics of the amino group $\mathrm{H}_{\mathrm{N}}$ nuclei in anions compared to zwitterion and protonated forms (S3-S5) are all compiled in one Supplementary Information (SI) pdf file. This information is available free of charge via the Internet at http://pubs.acs.org. Files with optimized geometries will be made available upon request. 


\section{References}

(1) Frank, A.; Möller, H. M.; Exner, T. E. Toward the Quantum Chemical Calculation of NMR Chemical Shifts of Proteins. 2. Level of Theory, Basis Set, and Solvents Model Dependence. J. Chem. Theory Comput. 2012, 8 (4), 1480-1492.

(2) Mulder, F. A. A.; Filatov, M. NMR Chemical Shift Data and Ab Initio Shielding Calculations: Emerging Tools for Protein Structure Determination. Chem Soc Rev 2010, 39 (2), 578-590.

(3) Hunter, C. A.; Packer, M. J.; Zonta, C. From Structure to Chemical Shift and Vice-Versa. Prog. Nucl. Magn. Reson. Spectrosc. 2005, 47 (1-2), 27-39.

(4) Wishart, D. S.; Bigam, C. G.; Holm, A.; Hodges, R. S.; Sykes, B. D. ${ }^{1} \mathrm{H},{ }^{13} \mathrm{C}$ and ${ }^{15} \mathrm{~N}$ Random Coil NMR Chemical Shifts of the Common Amino Acids. I. Investigations of Nearest-Neighbor Effects. J. Biomol. NMR 1995, 5 (1), 67-81.

(5) Williamson, M. P. Using Chemical Shift Perturbation to Characterise Ligand Binding. Prog. Nucl. Magn. Reson. Spectrosc. 2013, 73, 1-16.

(6) Carvalho, A. T. P.; Barrozo, A.; Doron, D.; Kilshtain, A. V.; Major, D. T.; Kamerlin, S. C. L. Challenges in Computational Studies of Enzyme Structure, Function and Dynamics. $J$. Mol. Graph. Model. 2014, 54, 62-79.

(7) Han, Y.-H.; Garron, M.-L.; Kim, H.-Y.; Kim, W.-S.; Zhang, Z.; Ryu, K.-S.; Shaya, D.; Xiao, Z.; Cheong, C.; Kim, Y. S.; et al. Structural Snapshots of Heparin Depolymerization by Heparin Lyase I. J. Biol. Chem. 2009, 284 (49), 34019-34027.

(8) Roggatz, C. C.; Lorch, M.; Hardege, J. D.; Benoit, D. M. Ocean Acidification Affects Marine Chemical Communication by Changing Structure and Function of Peptide Signalling Molecules. Glob. Change Biol. 2016, 22 (12), 3914-3926.

(9) Facelli, J. C. Calculations of Chemical Shieldings: Theory and Applications. Concepts Magn. Reson. 2004, $20 A$ (1), 42-69.

(10) Lodewyk, M. W.; Siebert, M. R.; Tantillo, D. J. Computational Prediction of ${ }^{1} \mathrm{H}$ and ${ }^{13} \mathrm{C}$ Chemical Shifts: A Useful Tool for Natural Product, Mechanistic, and Synthetic Organic Chemistry. Chem. Rev. 2012, 112 (3), 1839-1862.

(11) Modeling Solvent Environments: Applications to Simulations of Biomolecules; Feig, M., Ed.; Wiley-VCH: Weinheim, 2010.

(12) Buckingham, A. D.; Schaefer, T.; Schneider, W. G. Solvent Effects in Nuclear Magnetic Resonance Spectra. J. Chem. Phys. 1960, 32 (4), 1227-1233.

(13) Cossi, M.; Crescenzi, O. Different Models for the Calculation of Solvent Effects on ${ }^{17} \mathrm{O}$ Nuclear Magnetic Shielding. J. Chem. Phys. 2003, 118 (19), 8863.

(14) Schaefer, T.; Schneider, W. G. On the Nature of Solvent Effects in the Proton Resonance Spectra of Unsaturated Ring Compounds. I. Substituted Benzenes. J. Chem. Phys. 1960, 32 (4), 1218.

(15) Miertuš, S.; Scrocco, E.; Tomasi, J. Electrostatic Interaction of a Solute with a Continuum. A Direct Utilizaion of AB Initio Molecular Potentials for the Prevision of Solvent Effects. Chem. Phys. 1981, 55 (1), 117-129.

(16) Onsager, L. Electric Moments of Molecules in Liquids. J. Am. Chem. Soc. 1936, 58 (8), 1486-1493.

(17) Foresman, J. B.; Keith, T. A.; Wiberg, K. B.; Snoonian, J.; Frisch, M. J. Solvent Effects. 5. Influence of Cavity Shape, Truncation of Electrostatics, and Electron Correlation on $\mathrm{Ab}$ Initio Reaction Field Calculations. J. Phys. Chem. 1996, 100 (40), 16098-16104.

(18) Klamt, A.; Schüürmann, G. COSMO: A New Approach to Dielectric Screening in Solvents with Explicit Expressions for the Screening Energy and Its Gradient. J Chem Soc Perkin Trans 2 1993, No. 5, 799-805. 
(19) Tomasi, J.; Mennucci, B.; Cances, E. The IEF Version of the PCM Solvation Method: An Overview of a New Method Addressed to Study Molecular Solutes at the QM Ab Initio Level. J. Mol. Struct. THEOCHEM 1999, 464 (1), 211-226.

(20) Klamt, A. Conductor-like Screening Model for Real Solvents: A New Approach to the Quantitative Calculation of Solvation Phenomena. J. Phys. Chem. 1995, 99 (7), 22242235.

(21) Kovalenko, A.; Hirata, F. Potentials of Mean Force of Simple Ions in Ambient Aqueous Solution. I. Three-Dimensional Reference Interaction Site Model Approach. J. Chem. Phys. 2000, 112 (23), 10391-10402.

(22) Bachrach, S. M. Computational Organic Chemistry; John Wiley \& Sons, 2007.

(23) Lewars, E. G. Some "Special" Topics: Solvation, Singlet Diradicals, A Note on Heavy Atoms and Transition Metals. In Computational Chemistry; Springer Netherlands: Dordrecht, 2011; pp 521-560.

(24) Kelly, C. P.; Cramer, C. J.; Truhlar, D. G. Adding Explicit Solvent Molecules to Continuum Solvent Calculations for the Calculation of Aqueous Acid Dissociation Constants. J. Phys. Chem. A 2006, 110 (7), 2493-2499.

(25) Hass, M. A. S.; Mulder, F. A. A. Contemporary NMR Studies of Protein Electrostatics. Annu. Rev. Biophys. 2015, 44 (1), 53-75.

(26) Boron, W. F.; Boulpaep, E. L. Medical Physiology: A Cellular and Molecular Approach, Second; Saunders Elsevier: Philadelphia, 2009.

(27) Dračínský, M.; Bouř, P. Computational Analysis of Solvent Effects in NMR Spectroscopy. J. Chem. Theory Comput. 2010, 6 (1), 288-299.

(28) Balabin, R. M. Experimental Thermodynamics of Free Glycine Conformations: The First Raman Experiment after Twenty Years of Calculations. Phys. Chem. Chem. Phys. 2012, $14(1), 99$.

(29) Noguera, M.; Rodrıguez-Santiago, L.; Sodupe, M.; Bertran, J. Protonation of Glycine, Serine and Cysteine. Conformations, Proton Affinities and Intrinsic Basicities. J. Mol. Struct. Theochem 2001, 537 (1), 307-318.

(30) Hanwell, M. D.; Curtis, D. E.; Lonie, D. C.; Vandermeersch, T.; Zurek, E.; Hutchison, G. R. Avogadro: An Advanced Semantic Chemical Editor, Visualization, and Analysis Platform. J. Cheminformatics 2012, 4 (1), 17.

(31) Rappé, A. K.; Casewit, C. J.; Colwell, K. S.; Goddard Iii, W. A.; Skiff, W. M. UFF, a Full Periodic Table Force Field for Molecular Mechanics and Molecular Dynamics Simulations. J. Am. Chem. Soc. 1992, 114 (25), 10024-10035.

(32) Godfrey, P. D.; Firth, S.; Hatherley, L. D.; Brown, R. D.; Pierlot, A. P. Millimeter-Wave Spectroscopy of Biomolecules: Alanine. J. Am. Chem. Soc. 1993, 115 (21), 9687-9691.

(33) Adamo, C.; Barone, V. Toward Reliable Density Functional Methods without Adjustable Parameters: The PBE0 Model. J. Chem. Phys. 1999, 110 (13), 6158-6170.

(34) Jensen, F. Polarization Consistent Basis Sets: Principles. J. Chem. Phys. 2001, 115 (20), 9113-9125.

(35) Jensen, F. Polarization Consistent Basis Sets. II. Estimating the Kohn-Sham Basis Set Limit. J. Chem. Phys. 2002, 116 (17), 7372-7379.

(36) Jensen, F. Polarization Consistent Basis Sets. III. The Importance of Diffuse Functions. $J$. Chem. Phys. 2002, 117 (20), 9234-9240.

(37) Neese, F.; Wennmohs, F.; Hansen, A.; Becker, U. Efficient, Approximate and Parallel Hartree-Fock and Hybrid DFT Calculations. A 'Chain-of-Spheres' Algorithm for the Hartree-Fock Exchange. Chem. Phys. 2009, 356 (1-3), 98-109. 
(38) Weigend, F.; Ahlrichs, R. Balanced Basis Sets of Split Valence, Triple Zeta Valence and Quadruple Zeta Valence Quality for H to Rn: Design and Assessment of Accuracy. Phys. Chem. Chem. Phys. 2005, 7 (18), 3297-3305.

(39) Grimme, S.; Antony, J.; Ehrlich, S.; Krieg, H. A Consistent and Accurate Ab Initio Parametrization of Density Functional Dispersion Correction (DFT-D) for the 94 Elements H-Pu. J. Chem. Phys. 2010, 132 (15), 154104-154104.

(40) Grimme, S.; Ehrlich, S.; Goerigk, L. Effect of the Damping Function in Dispersion Corrected Density Functional Theory. J. Comput. Chem. 2011, 32 (7), 1456-1465.

(41) Kutzelnigg, W.; Fleischer, U.; Schindler, M. The IGLO-Method: Ab-Initio Calculation and Interpretation of NMR Chemical Shifts and Magnetic Susceptibilities. In Deuterium and Shift Calculation; NMR Basic Principles and Progress; Springer Berlin Heidelberg, 1991; Vol. 23, pp 165-262.

(42) Neese, F. The ORCA Program System. Wiley Interdiscip Rev Comput Mol Sci 2012, 2, $73-$ 78.

(43) Benassi, E. Benchmarking of Density Functionals for a Soft but Accurate Prediction and Assignment of ${ }^{1} \mathrm{H}$ and ${ }^{13} \mathrm{C}$ NMR Chemical Shifts in Organic and Biological Molecules. $J$. Comput. Chem. 2017, 38 (2), 87-92.

(44) Baggioli, A.; Crescenzi, O.; Field, M. J.; Castiglione, F.; Raos, G. Computational ${ }^{17} \mathrm{O}-$ NMR Spectroscopy of Organic Acids and Peracids: Comparison of Solvation Models. Phys. Chem. Chem. Phys. 2013, 15 (4), 1130.

(45) Benzi, C.; Improta, R.; Scalmani, G.; Barone, V. Quantum Mechanical Study of the Conformational Behavior of Proline and 4R-Hydroxyproline Dipeptide Analogues in Vacuum and in Aqueous Solution. J. Comput. Chem. 2002, 23 (3), 341-350.

(46) Improta, R.; Mele, F.; Crescenzi, O.; Benzi, C.; Barone, V. Understanding the Role of Stereoelectronic Effects in Determining Collagen Stability. 2. A Quantum Mechanical/Molecular Mechanical Study of (Proline-Proline-Glycine) ${ }_{n}$ Polypeptides. $J$. Am. Chem. Soc. 2002, 124 (26), 7857-7865.

(47) Benzi, C.; Crescenzi, O.; Pavone, M.; Barone, V. Reliable NMR Chemical Shifts for Molecules in Solution by Methods Rooted in Density Functional Theory. Magn. Reson. Chem. 2004, 42 (S1), S57-S67.

(48) Krishnan, R.; Binkley, J. S.; Seeger, R.; Pople, J. A. Self-consistent Molecular Orbital Methods. XX. A Basis Set for Correlated Wave Functions. J. Chem. Phys. 1980, 72 (1), 650-654.

(49) McLean, A. D.; Chandler, G. S. Contracted Gaussian Basis Sets for Molecular Calculations. I. Second Row Atoms, $Z=11-18$. J. Chem. Phys. 1980, 72 (10), 5639-5648.

(50) Bachrach, S. M. Microsolvation of Glycine: A DFT Study. J. Phys. Chem. A 2008, 112 (16), 3722-3730.

(51) Jensen, F. Basis Set Convergence of Nuclear Magnetic Shielding Constants Calculated by Density Functional Methods. J. Chem. Theory Comput. 2008, 4 (5), 719-727.

(52) Lide, D. R. CRC Handbook of Chemistry and Physics, 85th ed.; CRC press, 2004.

(53) Piotto, M.; Saudek, V.; Sklenáŕ, V. Gradient-Tailored Excitation for Single-Quantum NMR Spectroscopy of Aqueous Solutions. J. Biomol. NMR 1992, 2 (6), 661-665.

(54) Sklenáŕ, V.; Piotto, M.; Leppik, R.; Saudek, V. Gradient-Tailored Water Suppression for ${ }^{1} \mathrm{H}^{-15} \mathrm{~N}$ HSQC Experiments Optimized to Retain Full Sensitivity. J. Magn. Reson. A 1993, 102, 241-245.

(55) Bryant, R. G. The NMR Time Scale. J Chem Educ 1983, 60 (11), 933.

(56) Nazarski, R. B.; Wałejko, P.; Witkowski, S. Multi-Conformer Molecules in Solutions: An NMR-Based DFT/MP2 Conformational Study of Two Glucopyranosides of a Vitamin E Model Compound. Org. Biomol. Chem. 2016, 14 (11), 3142-3158. 
(57) Tantillo, D. J.; Lodewyk, M. W.; Siebert, M. R.; Rablen, P. R.; Bally, T. CHESHIRE CCAT - the Chemical Shift Repository for computed NMR scaling factors, with Coupling Constants Added Too http://cheshirenmr.info (accessed Nov 1, 2017).

(58) Gaussian Online Manual: NMR Calculations http://gaussian.com/nmr/.

(59) Sousa, S. F.; Fernandes, P. A.; Ramos, M. J. Gas-Phase Geometry Optimization of Biological Molecules as a Reasonable Alternative to a Continuum Environment Description: Fact, Myth, or Fiction? J. Phys. Chem. A 2009, 113 (52), 14231-14236.

(60) Frisch, M. J.; Trucks, G. W.; Schlegel, H. B.; Scuseria, G. E.; Robb, M. A.; Cheeseman, J. R.; Scalmani, G.; Barone, V.; Mennucci, B.; Petterson, G. A.; et al. Gaussian 09 Revision B.01.

(61) Ditchfield, R. Self-Consistent Perturbation Theory of Diamagnetism: I. A Gauge-Invariant LCAO Method for N.M.R. Chemical Shifts. Mol. Phys. 1974, 27 (4), 789-807.

(62) Wolinski, K.; Hinton, J. F.; Pulay, P. Efficient Implementation of the Gauge-Independent Atomic Orbital Method for NMR Chemical Shift Calculations. J. Am. Chem. Soc. 1990, 112 (23), 8251-8260.

(63) Sefzik, T. H.; Turco, D.; Iuliucci, R. J.; Facelli, J. C. Modeling NMR Chemical Shift: A Survey of Density Functional Theory Approaches for Calculating Tensor Properties. $J$. Phys. Chem. A 2005, 109 (6), 1180-1187.

(64) Kupka, T.; Stachów, M.; Nieradka, M.; Kaminsky, J.; Pluta, T. Convergence of Nuclear Magnetic Shieldings in the Kohn-Sham Limit for Several Small Molecules. J. Chem. Theory Comput. 2010, 6 (5), 1580-1589.

(65) Sure, R.; Grimme, S. Corrected Small Basis Set Hartree-Fock Method for Large Systems. J. Comput. Chem. 2013, 34 (19), 1672-1685.

(66) Jeffrey, G. A. An Introduction to Hydrogen Bonding; Oxford University Press: New York, 1997.

(67) Moon, S.; Case, D. A. A New Model for Chemical Shifts of Amide Hydrogens in Proteins. J. Biomol. NMR 2007, 38 (2), 139-150.

(68) Caldeweyher, E.; Bannwarth, C.; Grimme, S. Extension of the D3 Dispersion Coefficient Model. J. Chem. Phys. 2017, 147 (3), 034112.

(69) Kim, J.-Y.; Ahn, D.-S.; Park, S.-W.; Lee, S. Gas Phase Hydration of Amino Acids and Dipeptides: Effects on the Relative Stability of Zwitterion vs. Canonical Conformers. RSC Adv. 2014, 4 (31), 16352-16361.

(70) Panuszko, A.; Adamczak, B.; Czub, J.; Gojło, E.; Stangret, J. Hydration of Amino Acids: FTIR Spectra and Molecular Dynamics Studies. Amino Acids 2015, 47 (11), 2265-2278.

(71) Pappas, C. G.; Tzakos, A. G.; Gerothanassis, I. P. On the Hydration State of Amino Acids and Their Derivatives at Different Ionization States: A Comparative Multinuclear NMR and Crystallographic Investigation. J. Amino Acids 2012, 2012, 1-11.

(72) Sun, J.; Bousquet, D.; Forbert, H.; Marx, D. Glycine in Aqueous Solution: Solvation Shells, Interfacial Water, and Vibrational Spectroscopy from Ab Initio Molecular Dynamics. J. Chem. Phys. 2010, 133 (11), 114508.

(73) Ramaekers, R.; Pajak, J.; Lambie, B.; Maes, G. Neutral and Zwitterionic Glycine. $\mathrm{H}_{2} \mathrm{O}$ Complexes: A Theoretical and Matrix-Isolation Fourier Transform Infrared Study. $J$. Chem. Phys. 2004, 120 (9), 4182-4193.

(74) Biedermannová, L.; Schneider, B. Structure of the Ordered Hydration of Amino Acids in Proteins: Analysis of Crystal Structures. Acta Crystallogr. D Biol. Crystallogr. 2015, 71 (11), 2192-2202.

(75) Hori, S.; Yamauchi, K.; Kuroki, S.; Ando, I. Proton NMR Chemical Shift Behavior of Hydrogen-Bonded Amide Proton of Glycine-Containing Peptides and Polypeptides as Studied by Ab Initio MO Calculation. Int. J. Mol. Sci. 2002, 3 (8), 907-913. 
(76) van Mourik, T. Density Functional Theory Reveals an Increase in the Amino H1 Chemical Shift in Guanine Due to Hydrogen Bonding with Water. J. Chem. Phys. 2006, 125 (19), 191101.

(77) Cordier, F.; Nisius, L.; Dingley, A. J.; Grzesiek, S. Direct Detection of $\mathrm{N}-\mathrm{H} \cdots \mathrm{O}=\mathrm{C}$ Hydrogen Bonds in Biomolecules by NMR Spectroscopy. Nat. Protoc. 2008, 3 (2), 235241.

(78) Merutka, G.; Dyson, H. J.; Wright, P. E. 'Random Coil' ${ }^{1} \mathrm{H}$ Chemical Shifts Obtained as a Function of Temperature and Trifluoroethanol Concentration for the Peptide Series GGXGG. J. Biomol. NMR 1995, 5 (1), 14-24.

(79) Exner, T. E.; Frank, A.; Onila, I.; Möller, H. M. Toward the Quantum Chemical Calculation of NMR Chemical Shifts of Proteins. 3. Conformational Sampling and Explicit Solvents Model. J. Chem. Theory Comput. 2012, 8 (11), 4818-4827.

(80) Flaig, D.; Maurer, M.; Hanni, M.; Braunger, K.; Kick, L.; Thubauville, M.; Ochsenfeld, C. Benchmarking Hydrogen and Carbon NMR Chemical Shifts at HF, DFT, and MP2 Levels. J. Chem. Theory Comput. 2014, 10 (2), 572-578.

(81) Auer, A. A.; Gauss, J.; Stanton, J. F. Quantitative Prediction of Gas-Phase ${ }^{13} \mathrm{C}$ Nuclear Magnetic Shielding Constants. J. Chem. Phys. 2003, 118 (23), 10407-10417.

(82) Rablen, P. R.; Pearlman, S. A.; Finkbiner, J. A Comparison of Density Functional Methods for the Estimation of Proton Chemical Shifts with Chemical Accuracy. J. Phys. Chem. A 1999, 103 (36), 7357-7363.

(83) Zaborsky, O. R.; Milliman, G. E. The Effect of Temperature on the Chemical Shifts of the Histidine Residues of Ribonuclease A. Biochim. Biophys. Acta BBA-Protein Struct. 1972, 271 (2), 274-278.

(84) Wishart, D. S.; Bigam, C. G.; Yao, J.; Abildgaard, F.; Dyson, H. J.; Oldfield, E.; Markley, J. L.; Sykes, B. D. ${ }^{1} \mathrm{H},{ }^{13} \mathrm{C}$ and ${ }^{15} \mathrm{~N}$ Chemical Shift Referencing in Biomolecular NMR. $J$. Biomol. NMR 1995, 6 (2), 135-140.

(85) Monajjemi, M.; Heshmat, M.; Aghaei, H.; Ahmadi, R.; Zare, K. Solvent Effect on ${ }^{14} \mathrm{~N}$ NMR Shielding of Glycine, Serine, Leucine, and Threonine: Comparison between Chemical Shifts and Energy versus Dielectric Constant. Bull. Chem. Soc. Ethiop. 2007, 21 (1).

(86) Degtyarenko, I. M.; Jalkanen, K. J.; Gurtovenko, A. A.; Nieminen, R. M. L-Alanine in a Droplet of Water: A Density-Functional Molecular Dynamics Study. J. Phys. Chem. B 2007, 111 (16), 4227-4234.

(87) Kwan, E. E.; Liu, R. Y. Enhancing NMR Prediction for Organic Compounds Using Molecular Dynamics. J. Chem. Theory Comput. 2015, 11 (11), 5083-5089.

(88) Ball, K. A.; Wemmer, D. E.; Head-Gordon, T. Comparison of Structure Determination Methods for Intrinsically Disordered Amyloid- $\beta$ Peptides. J. Phys. Chem. B 2014, 118 (24), 6405-6416.

(89) Dračínský, M.; Möller, H. M.; Exner, T. E. Conformational Sampling by Ab Initio Molecular Dynamics Simulations Improves NMR Chemical Shift Predictions. J. Chem. Theory Comput. 2013, 9 (8), 3806-3815.

(90) Markland, T. E.; Ceriotti, M. Nuclear Quantum Effects Enter the Mainstream. Nat. Rev. Chem. 2018, 2 (3), 0109.

(91) Dračínský, M.; Hodgkinson, P. Effects of Quantum Nuclear Delocalisation on NMR Parameters from Path Integral Molecular Dynamics. Chem. - Eur. J. 2014, 20 (8), 22012207.

(92) Shiga, M.; Suzuki, K.; Tachikawa, M. The Chemical Shift of Deprotonated Water Dimer: Ab Initio Path Integral Simulation. J. Chem. Phys. 2010, 132 (11), 114104. 